Proyecciones Journal of Mathematics

Vol. 36, $\mathrm{N}^{o}$ 1, pp. 149-186, March 2017.

Universidad Católica del Norte

Antofagasta - Chile

\title{
Strong right fractional calculus for Banach space valued functions
}

\author{
George A. Anastassiou \\ University of Memphis, U. S. A. \\ Received: October 2016. Accepted : November 2016
}

\begin{abstract}
We present here a strong right fractional calculus theory for Banach space valued functions of Caputo type. Then we establish many right fractional Bochner integral inequalities of various types.

2010 AMS Subject Classification : 26A33, 26D10, 26D15, 46B25.

Key Words and Phrases : Right Fractional derivative, Right Fractional Taylor's formula, Banach space valued functions, integral inequalities, Hausdorff measure, Bochner integral.
\end{abstract}




\section{Introduction}

Here we use extensively the Bochner integral for Banach space valued functions, which is a direct generalization of Lebesgue integral to this case. The reader may read about Bochner integral and its properties from [2], [5], [6], [8], [10], [11] and [12].

Using Bochner integral properties and the great article [12], we develop a right Caputo type strong fractional theory for the first time in the literature, which is the direct analog of the real one, but now dealing with Banach space valued functions.

In the literature there are very few articles about the left weak fractional theory of Banach space valued functions with one of the best [1]. None exists about the right one.

However we found the left weak theory, using Pettis integral and functionals, complicated, less clear, dificult and unnecessary.

With this article we try to simplify matters and put the related right theory on its natural grounds and resemble the theory on real numbers.

We define the right Riemann-Liouville fractional Bochner integral operator, see Definition 2, and we prove the right commutative semigroup property, see Theorem 7 .

We use the general Fundamental theorem of calculus for Bochner integration, see Theorem 10 here, from [12].

Based on the last we produce a related reverse general Taylor's formula for Banach valued functions.

We introduce then the right Caputo type fractional derivative in our setting, see Definition 13. Then we are able to produce the related right fractional Taylor's formula in Banach space setting, which involves the Hausdorff measure. With this developed machinery we derive right fractional: Ostrowski type inequalities, Poincaré and Sobolev types, Opial type, Hilbert-Pachpatte type, and Landau type inequalities.

All these right fractional inequalities for Banach space valued functions are using always the Hausdorff measure. We cover these inequalities to all possible directions, acting at the introductory basic level, which leaves big room for expansions later.

\section{Main Results}

We mention 
Definition 1. Let $U \subseteq \mathbf{R}$ be an interval, and $X$ is a Banach space, we denote by $L_{1}(U, X)$ the Bochner integrable functions from $U$ into $X$.

We need

Definition 2. Let $\alpha>0,[a, b] \subset \mathbf{R}, X$ is a Banach space, and $f \in$ $L_{1}([a, b], X)$. The Bochner integral operator

$$
\left(I_{b-}^{\alpha} f\right)(x):=\frac{1}{\Gamma(\alpha)} \int_{x}^{b}(z-x)^{\alpha-1} f(z) d z,
$$

$\forall x \in[a, b]$, where $\Gamma$ is the gamma function, is called the RiemannLiouville right fractional Bochner integral operator of order $\alpha$.

For $\alpha=0$, we set $I_{b-}^{0}:=I$ (the identity operator).

We need

Theorem 3. Let $f \in L_{1}([a, b], X), \alpha>0$. Then $\left(I_{b-}^{\alpha} f\right)(x)$ exists almost everywhere on $[a, b]$, and $I_{b-}^{\alpha} f \in L_{1}([a, b], X)$.

Proof. Define $k: \Omega:=[a, b]^{2} \rightarrow \mathbf{R}$ by $k(z, x)=(z-x)_{+}^{\alpha-1}$, that is

$$
k(z, x)=\left\{\begin{array}{l}
(z-x)^{\alpha-1}, \text { if } a \leq x \leq z \leq b \\
0, \text { if } a \leq z \leq x \leq b .
\end{array}\right.
$$

Then $k$ is measurable on $\Omega$, and we have $\int_{a}^{b} k(z, x) d x=\int_{a}^{z} k(z, x) d x+$ $\int_{z}^{b} k(z, x) d x=$

$$
\int_{z}^{z}(z-x)^{\alpha-1} d x=\frac{(z-a)^{\alpha}}{\alpha} .
$$

Let $\chi_{[a, b]}(x)$ be the characteristic function, $x \in \mathbf{R}$. By [10], p. 101, Theorem 5.4, we get that $f(z) \chi_{[a, b]}(x)=f(z)$ on $[a, b]^{2}$, is strongly (Bochner) measurable function on $[a, b]^{2}$. Clearly $k(z, x)$ is finite a.e on $[a, b]^{2}$, and it is a real valued measurable function. By [7], p. 88, we get now that

$$
\mathrm{k}(z, x) f(z) \chi_{[a, b]}(x)=k(z, x) f(z) \text { on }[a, b]^{2}
$$

is strongly (Bochner) measurable function there.

Next we work on the repeated integral

$$
\begin{gathered}
\int_{a}^{b}\left(\int_{a}^{b} k(z, x)\|f(z)\| d x\right) d z=\int_{a}^{b}\|f(z)\|\left(\int_{a}^{b} k(z, x) d x\right) d z= \\
\int_{a}^{b}\|f(z)\| \frac{(z-a)^{\alpha}}{\alpha} d z \leq \frac{(b-a)^{\alpha}}{\alpha} \int_{a}^{b}\|f(z)\| d z
\end{gathered}
$$




$$
=\frac{(b-a)^{\alpha}}{\alpha}\|f\|_{L_{1}([a, b], X)}<\infty .
$$

Therefore the function $H: \Omega \rightarrow X$ such that $H(z . x):=k(z, x) f(z)$ is Bochner integrable over $\Omega$ by Tonelli's theorem, see [10], p. 100, Theorem 5.2 .

Hence by Fubini's theorem, [10], p. 93, Theorem 2, we obtain that $\int_{a}^{b} k(z, x) f(z) d z$ is a Bochner integrable function on $[a, b]$, as a function of $x \in[a, b]$. That is $\left(I_{b-}^{\alpha} f\right)(x)=\frac{1}{\Gamma(\alpha)} \int_{x}^{b}(z-x)^{\alpha-1} f(z) d z$ is Bochner integrable on $[a, b]$, and exists a.e. on $[a, b]$.

We further present and need

Lemma 4. Let $\alpha \geq 1$ and $f \in L_{1}([a, b], X), X$ a Banach space. Then $I_{b-}^{\alpha} f \in C([a, b], X)$.

Proof. (i) Case of $\alpha=1$. We have that

$$
\left(I_{b-}^{1} f\right)(x)=\int_{x}^{b} f(z) d z .
$$

Let $x, y \in[a, b]: x \geq y$ and $x \rightarrow y$. We observe that

$$
\begin{gathered}
\left\|\left(I_{b-}^{1} f\right)(x)-\left(I_{b-}^{1} f\right)(y)\right\|=\left\|\int_{x}^{b} f(z) d z-\int_{y}^{b} f(z) d z\right\|([5])= \\
\left\|\int_{x}^{b} f(z) d z-\int_{y}^{x} f(z) d z-\int_{x}^{b} f(z) d z\right\|=\left\|\int_{y}^{x} f(z) d z\right\| \leq \\
\int_{y}^{x}\|f(z)\| d z=\int_{x}^{b}\|f(z)\| d z-\int_{y}^{b}\|f(z)\| d z \rightarrow 0,
\end{gathered}
$$

because $\int_{x}^{b}\|f(z)\| d z$ is continuous in $x \in[a, b]$.

(ii) Case of $\alpha>1$. Let $x, y \in[a, b]: x \geq y$ and $x \rightarrow y$.

We observe that

$$
\begin{gathered}
\left\|I_{b-}^{\alpha} f(x)-I_{b-}^{\alpha} f(y)\right\|= \\
\frac{1}{\Gamma(\alpha)}\left\|\int_{x}^{b}(\zeta-x)^{\alpha-1} f(\zeta) d \zeta-\int_{y}^{b}(\zeta-y)^{\alpha-1} f(\zeta) d \zeta\right\|([5])=
\end{gathered}
$$


$\frac{1}{\Gamma(\alpha)}\left\|\int_{x}^{b}(\zeta-x)^{\alpha-1} f(\zeta) d \zeta-\int_{y}^{x}(\zeta-y)^{\alpha-1} f(\zeta) d \zeta-\int_{x}^{b}(\zeta-y)^{\alpha-1} f(\zeta) d \zeta\right\|$

(see [2], p. 426, Theorem 11.43)

$$
\begin{aligned}
& \leq \frac{1}{\Gamma(\alpha)}\left[\int_{x}^{b}\left|(\zeta-x)^{\alpha-1}-(\zeta-y)^{\alpha-1}\right|\|f(\zeta)\| d \zeta+\int_{y}^{x}(\zeta-y)^{\alpha-1}\|f(\zeta)\| d \zeta\right] \\
& \leq \frac{1}{\Gamma(\alpha)}\left[\int_{x}^{b}\left|(\zeta-x)^{\alpha-1}-(\zeta-y)^{\alpha-1}\right|\|f(\zeta)\| d \zeta+(x-y)^{\alpha-1}\|f\|_{L_{1}([a, b], X)}\right] .
\end{aligned}
$$

As $x \rightarrow y$ we get $(\zeta-x)^{\alpha-1} \rightarrow(\zeta-y)^{\alpha-1}$, thus

$$
\left|(\zeta-x)^{\alpha-1}-(\zeta-y)^{\alpha-1}\right| \rightarrow 0,
$$

and also

$$
\left|(\zeta-x)^{\alpha-1}-(\zeta-y)^{\alpha-1}\right| \leq 2(b-a)^{\alpha-1}
$$

Thus

$$
\left|(\zeta-x)^{\alpha-1}-(\zeta-y)^{\alpha-1}\right|\|f(\zeta)\| \leq 2(b-a)^{\alpha-1}\|f(\zeta)\| \in L_{1}([a, b], X),
$$

and also $\left|(\zeta-x)^{\alpha-1}-(\zeta-y)^{\alpha-1}\right|\|f(\zeta)\| \rightarrow 0$ as $x \rightarrow y$, for almost all $\zeta \in[a, b]$.

Therefore by Dominated Convergence Theorem we conclude, as $x \rightarrow y$, that

$$
\int_{x}^{b}\left|(\zeta-x)^{\alpha-1}-(\zeta-y)^{\alpha-1}\right|\|f(\zeta)\| d \zeta \rightarrow 0 .
$$

Consequently,

$$
\left\|I_{b-}^{\alpha} f(x)-I_{b-}^{\alpha} f(y)\right\| \rightarrow 0 \text { as } x \rightarrow y .
$$

Therefore $I_{b-}^{\alpha} f \in C([a, b], X)$.

We give 
Theorem 5. Here $[a, b] \subset \mathbf{R}, X$ is a Banach space, $F:[a, b] \rightarrow X$. Let $r>0, F \in L_{\infty}([a, b], X)$, and the Bochner integral

$$
G(s):=\int_{s}^{b}(t-s)^{r-1} F(t) d t,
$$

all $s \in[a, b]$. Then $G \in A C([a, b], X)$ (absolutely continuous functions) for $r \geq 1$ and $G \in C([a, b], X)$ for $r \in(0,1)$.

Proof. Denote by $\|F\|_{\infty}:=\|F\|_{L_{\infty}([a, b], X)}:=t \in[a, b] \operatorname{es} \sup \|F(t)\|_{X}<$ $+\infty$. Hence $F \in L_{1}([a, b], X)$.

By [7], p. 88, $(t-s)^{r-1} F(t)$ is a strongly measurable function in $t$, $t \in[s, b], s \in[a, b]$. So that $(t-s)^{r-1} F(t) \in L_{1}([s, b], X)$, see [6].

(1) Case $r \geq 1$. We use the definition of absolute continuity. So for every $\varepsilon>0$ we need $\delta>0$ : whenever $\left(a_{i}, b_{i}\right), i=1, \ldots, n$, are disjoint subintervals of $[a, b]$, then

$$
\sum_{i=1}^{n}\left(b_{i}-a_{i}\right)<\delta \Rightarrow \sum_{i=1}^{n}\left\|G\left(b_{i}\right)-G\left(a_{i}\right)\right\|<\varepsilon
$$

If $\|F\|_{\infty}=0$, then $G(s)=0$, for all $s \in[a, b]$, the trivial case and all fulfilled.

So we assume $\|F\|_{\infty} \neq 0$. Hence we have (see [5])

$$
\begin{gathered}
G\left(b_{i}\right)-G\left(a_{i}\right)=\int_{b_{i}}^{b}\left(t-b_{i}\right)^{r-1} F(t) d t-\int_{a_{i}}^{b}\left(t-a_{i}\right)^{r-1} F(t) d t= \\
\int_{b_{i}}^{b}\left(t-b_{i}\right)^{r-1} F(t) d t-\int_{a_{i}}^{b_{i}}\left(t-a_{i}\right)^{r-1} F(t) d t-\int_{b_{i}}^{b}\left(t-a_{i}\right)^{r-1} F(t) d t=
\end{gathered}
$$

(see [2], p. 426, Theorem 11.43)

$$
\int_{b_{i}}^{b}\left(\left(t-b_{i}\right)^{r-1}-\left(t-a_{i}\right)^{r-1}\right) F(t) d t-\int_{a_{i}}^{b_{i}}\left(t-a_{i}\right)^{r-1} F(t) d t
$$

Call

$$
I_{i}:=\int_{b_{i}}^{b}\left|\left(t-b_{i}\right)^{r-1}-\left(t-a_{i}\right)^{r-1}\right| d t
$$


Thus

$$
\left\|G\left(b_{i}\right)-G\left(a_{i}\right)\right\| \leq\left[I_{i}+\frac{\left(b_{i}-a_{i}\right)^{r}}{r}\right]\|F\|_{\infty}:=T_{i} .
$$

If $r=1$, then $I_{i}=0$, and

$$
\left\|G\left(b_{i}\right)-G\left(a_{i}\right)\right\| \leq\|F\|_{\infty}\left(b_{i}-a_{i}\right)
$$

for all $i:=1, \ldots, n$.

If $r>1$, then because $\left[\left(t-a_{i}\right)^{r-1}-\left(t-b_{i}\right)^{r-1}\right] \geq 0$ for all $t \in\left[b_{i}, b\right]$, we find

$$
\mathrm{I}_{i}=\int_{b_{i}}^{b}\left(\left(t-a_{i}\right)^{r-1}-\left(t-b_{i}\right)^{r-1}\right) d t=\frac{\left(b-a_{i}\right)^{r}-\left(b_{i}-a_{i}\right)^{r}-\left(b-b_{i}\right)^{r}}{r}
$$

$$
=\frac{r(b-\xi)^{r-1}\left(b_{i}-a_{i}\right)-\left(b_{i}-a_{i}\right)^{r}}{r}, \text { for some } \xi \in\left(a_{i}, b_{i}\right)
$$

Therefore, it holds

$$
I_{i} \leq \frac{r(b-a)^{r-1}\left(b_{i}-a_{i}\right)-\left(b_{i}-a_{i}\right)^{r}}{r}
$$

and

$$
\left(I_{i}+\frac{\left(b_{i}-a_{i}\right)^{r}}{r}\right) \leq(b-a)^{r-1}\left(b_{i}-a_{i}\right)
$$

That is

$$
T_{i} \leq\|F\|_{\infty}(b-a)^{r-1}\left(b_{i}-a_{i}\right)
$$

so that

$$
\left\|G\left(b_{i}\right)-G\left(a_{i}\right)\right\| \leq\|F\|_{\infty}(b-a)^{r-1}\left(b_{i}-a_{i}\right), \text { for all } i=1, \ldots, n \text {. }
$$

So in the case of $r=1$, and by choosing $\delta:=\frac{\varepsilon}{\|F\|_{\infty}}$, we get

$$
\sum_{i=1}^{n}\left\|G\left(b_{i}\right)-G\left(a_{i}\right)\right\| \leq\|F\|_{\infty}\left(\sum_{i=1}^{n}\left(b_{i}-a_{i}\right)\right) \leq\|F\|_{\infty} \delta=\varepsilon
$$

proving for $r=1$ that $G$ is absolutely continuous. In the case of $r>1$, and by choosing $\delta:=\frac{\varepsilon}{\|F\|_{\infty}(b-a)^{r-1}}$, we get 


$$
\begin{gathered}
\sum_{i=1}^{n}\left\|G\left(b_{i}\right)-G\left(a_{i}\right)\right\| \leq\|F\|_{\infty}(b-a)^{r}\left(\sum_{i=1}^{n}\left(b_{i}-a_{i}\right)\right) \\
\leq\|F\|_{\infty}(b-a)^{r-1} \delta=\varepsilon,
\end{gathered}
$$

proving for $r>1$ that $G$ is absolutely continuous again.

(2) Case of $0<r<1$. Let $a_{i *}, b_{i *} \in[a, b]: a_{i *} \leq b_{i *}$. Then $\left(t-a_{i *}\right)^{r-1} \leq$ $\left(t-b_{i *}\right)^{r-1}$, for all $t \in\left[b_{i}, b\right]$. Then

$$
\mathrm{I}_{i *}=\int_{b_{i *}}^{b}\left(\left(t-b_{i *}\right)^{r-1}-\left(t-a_{i *}\right)^{r-1}\right) d t=\frac{\left(b-b_{i *}\right)^{r}}{r}-
$$

$$
\left(\frac{\left(b-a_{i *}\right)^{r}-\left(b_{i *}-a_{i *}\right)^{r}}{r}\right) \leq \frac{\left(b_{i *}-a_{i *}\right)^{r}}{r}
$$

by $\left(b-b_{i *}\right)^{r}-\left(b-a_{i *}\right)^{r}<0$.

Therefore

$$
I_{i *} \leq \frac{\left(b_{i *}-a_{i *}\right)^{r}}{r}
$$

and

$$
T_{i *} \leq \frac{2\left(b_{i *}-a_{i *}\right)^{r}}{r}\|F\|_{\infty}
$$

proving that

$$
\left\|G\left(b_{i *}\right)-G\left(a_{i *}\right)\right\| \leq\left(\frac{2\|F\|_{\infty}}{r}\right)\left(b_{i *}-a_{i *}\right)^{r},
$$

which proves that $G$ is continuous.

This completes the proof.

We make

Remark 6. Let $[a, b] \subset \mathbf{R}$ and $(X,\|\cdot\|)$ a Banach space. Let also $f$ : $[a, b] \rightarrow X$. If $f$ is continuous, i.e. $f \in C([a, b], X)$, then $f$ is strongly measurable, by [8], Corollary 2.3, p. 5.

Furthermore $f([a, b]) \subseteq X$ is compact, thus it is closed and bounded, hence $f$ is bounded, i.e. $\|f(t)\| \leq M, \forall t \in[a, b], M>0$.

Let $x_{n}, x \in[a, b]: x_{n} \rightarrow x$, as $n \rightarrow \infty$, then $f\left(x_{n}\right) \rightarrow f(x)$ in $\|\cdot\|$, that is $\left|\left\|f\left(x_{n}\right)\right\|-\|f(x)\|\right| \leq\left\|f\left(x_{n}\right)-f(x)\right\| \rightarrow 0$, proving $\|f\|$ is continuous, hence bounded, so that $\|f\|_{L_{\infty}([a, b], X)}:=t \in[a, b] \operatorname{essup}\|f(t)\|<+\infty$, that is $f \in L_{\infty}([a, b], X)$, and hence $f \in L_{1}([a, b], X)$. Consequently, $f$ is Bochner integrable ([2], p. 426), given that $f$ is continuous. 
For the last we used the fact:

$$
\int_{[a, b]}\|f(t)\| d t \leq\|f\|_{L_{\infty}([a, b], X)}(b-a)<+\infty,
$$

proving that $f \in L_{1}([a, b], X)$.

Also, clearly, absolute continuity of $f:[a, b] \rightarrow X$, implies uniform continuity and continuity of $f$.

We also have

Theorem 7. Let $\alpha, \beta \geq 0, f \in L_{1}([a, b], X)$. Then

$$
I_{b-}^{\alpha} I_{b-}^{\beta} f=I_{b-}^{\alpha+\beta} f=I_{b-}^{\beta} I_{b-}^{\alpha} f,
$$

valid almost everywhere on $[a, b]$.

If additionally $f \in C([a, b], X)$ or $\alpha+\beta \geq 1$, then we have identity true on all of $[a, b]$.

Proof. Since $I_{b-}^{0}:=I$ (the identity operator), if $\alpha=0$ or $\beta=0$ or both are zero, then the statement of the theorem is trivially true. So we assume $\alpha, \beta>0$.

We observe that

$$
I_{b-}^{\alpha} I_{b-}^{\beta} f(x)=\frac{1}{\Gamma(\alpha) \Gamma(\beta)} \int_{x}^{b}(t-x)^{\alpha-1}\left(\int_{t}^{b}(\tau-t)^{\beta-1} f(\tau) d \tau\right) d t
$$

$$
=\frac{1}{\Gamma(\alpha) \Gamma(\beta)} \int_{x}^{b} \int_{x}^{b} \chi_{[t, b]}(\tau)(t-x)^{\alpha-1}(\tau-t)^{\beta-1} f(\tau) d \tau d t .
$$

The above integrals in (2.26) exist a.e. on $[a, b]$. So if $I_{b-}^{\alpha} I_{b-}^{\beta} f(x), I_{b-}^{\alpha+\beta} f(x)$ exist we can apply Fubini's theorem, see Theorem 2, p. 93, [10], to interchange the order of integration and obtain

$$
I_{b-}^{\alpha} I_{b-}^{\beta} f(x)=\frac{1}{\Gamma(\alpha) \Gamma(\beta)} \int_{x}^{b}\left(\int_{x}^{\tau}(t-x)^{\alpha-1}(\tau-t)^{\beta-1} f(\tau) d t\right) d \tau=
$$




$$
\begin{gathered}
\frac{1}{\Gamma(\alpha) \Gamma(\beta)} \int_{x}^{b} f(\tau)\left(\int_{x}^{\tau}(\tau-t)^{\beta-1}(t-x)^{\alpha-1} d t\right) d \tau= \\
\frac{1}{\Gamma(\alpha) \Gamma(\beta)} \int_{x}^{b} f(\tau) \frac{\Gamma(\alpha) \Gamma(\beta)}{\Gamma(\alpha+\beta)}(\tau-x)^{\alpha+\beta-1} d \tau= \\
\frac{1}{\Gamma(\alpha+\beta)} \int_{x}^{b} f(\tau)(\tau-x)^{(\alpha+\beta)-1} d \tau=I_{b-}^{\alpha+\beta} f(x) .
\end{gathered}
$$

That is

$$
I_{b-}^{\alpha} I_{b-}^{\beta} f(x)=I_{b-}^{\alpha+\beta} f(x)
$$

true a.e. on $[a, b]$.

By Theorem 5 and Remark 6 , if $f \in C([a, b], X)$, then $I_{b-}^{\beta} f \in C([a, b], X)$, therefore $I_{b-}^{\alpha} I_{b-a}^{\beta} f \in C([a, b], X)$ and $I_{b-}^{\alpha+\beta} f \in C([a, b], X)$.

Since in (29.) two continuous functions coincide a.e., the must be equal everywhere.

At last, if $f \in L_{1}([a, b], X)$ and $\alpha+\beta \geq 1$, we get $I_{b-}^{\alpha+\beta} f \in C([a, b], X)$ by Lemma 14. Hence, since $I_{b-}^{\alpha+\beta} f(x)$ is defined and existing for any $x \in$ $[a, b]$, by Fubini's theorem as before, equals to $I_{b-}^{\alpha} I_{b-}^{\beta} f(x)$, for all $x \in[a, b]$, proving the claim.

The algebraic version of previous theorem follows:

Theorem 8. The Bochner integral operators $\left\{I_{b-}^{\alpha}: L_{1}([a, b], X) \rightarrow L_{1}([a, b], X) ; \quad \alpha>0\right\}$ make a commutative semigroup with respect to composition. The identity operator $I_{b-}^{0}=I$ is the neutral element of this semigroup.

We need

Definition 9. (see [12]) A definition of the Hausdorff measure $h_{\alpha}$ goes as follows: if $(T, d)$ is a metric space, $A \subseteq T$ and $\delta>0$, let $\Lambda(A, \delta)$ be the set of all arbitrary collections $(C)_{i}$ of subsets of $T$, such that $A \subseteq \cup_{i} C_{i}$ and $\operatorname{diam}\left(C_{i}\right) \leq \delta$ (diam =diameter) for every $i$. Now, for every $\alpha>0$ define

$$
h_{\alpha}^{\delta}(A):=\inf \left\{\sum\left(\operatorname{diam} C_{i}\right)^{\alpha} \mid\left(C_{i}\right)_{i} \in \Lambda(A, \delta)\right\} .
$$

Then there exists $\delta \rightarrow 0 \lim h_{\alpha}^{\delta}(A)=\delta>0 \sup h_{\alpha}^{\delta}(A)$, and $h_{\alpha}(A):=$ $\delta \rightarrow 0 \lim h_{\alpha}^{\delta}(A)$ gives an outer measure on the power set $P(T)$, which is 
countably additive on the $\sigma$-field of all Borel subsets of T. If $T=\mathbf{R}^{n}$, then the Hausdorff measure $h_{n}$, restricted to the $\sigma$-field of the Borel subsets of $\mathbf{R}^{n}$, equals the Lebesgue measure on $\mathbf{R}^{n}$ up to a constant multiple. In particular, $h_{1}(C)=\mu(C)$ for every Borel set $C \subseteq \mathbf{R}$, where $\mu$ is the Lebesgue measure.

We will use the following spectacular result

Theorem 10. ([12]) (Fundamental Theorem of Calculus for Bochner integration)

Suppose that for the given function $f:[a, b] \rightarrow X$, there exists $F$ : $[a, b] \rightarrow X$, which is continuous, the derivative $F^{\prime}(t)$ exists and $F^{\prime}(t)=$ $f(t)$ outside a $\mu$-null Borel set $B \subseteq[a, b]$ such that $h_{1}(F(B))=0$.

Then $f$ is $\mu$-measurable (i.e. strongly measurable), and if we assume the Bochner integrability of $f$,

$$
F(b)-F(a)=\int_{a}^{b} f(t) d t .
$$

Notice here that the derivatives of a function $f:[a, b] \rightarrow X$, where $X$ is a Banach space, are defined exactly as the numerical ones, see for definitions and properties, [11], pp. 83-86, and p. 93, that is they are strong derivatives.

Notation 11. Let $f \in L_{1}([a, b], X)$. We denote by

$$
\int_{b}^{a} f(t) d t=-\int_{a}^{b} f(t) d t
$$

We will use Theorem 10 to give a general Taylor's formula for Banach space valued functions with a Bochner integral remainder.

Theorem 12. Let $n \in \mathbf{N}$ and $f \in C^{n-1}([a, b], X)$, where $[a, b] \subset \mathbf{R}$ and $X$ is a Banach space. Set

$$
F(x):=\sum_{i=0}^{n-1} \frac{(a-x)^{i}}{i !} f^{(i)}(x), x \in[a, b] .
$$

Assume that $f^{(n)}$ exists outside a $\mu$-null Borel set $B \subseteq[a, b]$ such that

$$
h_{1}(F(B))=0 .
$$

We further assume the Bochner integrability of $f^{(n)}$. Then 


$$
f(a)=\sum_{i=0}^{n-1} \frac{(a-b)^{i}}{i !} f^{(i)}(b)+\frac{1}{(n-1) !} \int_{b}^{a}(a-t)^{n-1} f^{(n)}(t) d t
$$

Proof. We get that $F \in C([a, b], X)$. Notice that $F(a)=f(a)$, and

$$
F(b)=\sum_{i=0}^{n-1} \frac{(a-b)^{i}}{i !} f^{(i)}(b) .
$$

Clearly $F^{\prime}$ exists outside of $B$. Infact it holds

$$
F^{\prime}(x)=\frac{(a-x)^{n-1}}{(n-1) !} f^{(n)}(x), \quad \forall x \in[a, b]-B .
$$

Also $F^{\prime}$ is Bochner integrable.

By Theorem 10 now we get that

$$
F(b)-F(a)=\int_{a}^{b} F^{\prime}(t) d t .
$$

That is, we have $\sum_{i=0}^{n-1} \frac{(a-b)^{i}}{i !} f^{(i)}(b)-f(a)=\int_{a}^{b} \frac{(a-t)^{n-1}}{(n-1) !} f^{(n)}(t) d t=$

$$
-\int_{b}^{a} \frac{(a-t)^{n-1}}{(n-1) !} f^{(n)}(t) d t
$$

proving (2.35).

We give

Definition 13. Let $[a, b] \subset \mathbf{R}, X$ be a Banach space, $\alpha>0, m:=\lceil\alpha\rceil$, (「· the ceiling of the number). We assume that $f^{(m)} \in L_{1}([a, b], X)$, where $f:[a, b] \rightarrow X$. We call the Caputo-Bochner right fractional derivative of order $\alpha$ :

$$
\left(D_{b-}^{\alpha} f\right)(x):=(-1)^{m} I_{b-}^{m-\alpha} f^{(m)}(x),
$$

i.e.

$$
\left(D_{b-}^{\alpha} f\right)(x):=\frac{(-1)^{m}}{\Gamma(m-\alpha)} \int_{x}^{b}(J-x)^{m-\alpha-1} f^{(m)}(J) d J, \quad \forall x \in[a, b] .
$$


We observe that $D_{b-}^{m} f(x)=(-1)^{m} f^{(m)}(x)$, for $m \in \mathbf{N}$, and $D_{b-}^{0} f(x)=f(x)$.

By Theorem $3\left(D_{b-}^{\alpha} f\right)(x)$ exists almost everywhere on $[a, b]$ and $\left(D_{b-}^{\alpha} f\right) \in L_{1}([a, b], X)$.

If $\left\|f^{(m)}\right\|_{L_{\infty}([a, b], X)}<\infty$, and $\alpha \notin \mathbf{N}$, then by Theorem 5 , $D_{b-}^{\alpha} f \in C([a, b], X)$, hence $\left\|D_{b-}^{\alpha} f\right\| \in C([a, b])$.

We make

Remark 14. (to Definition 13) We notice that (by Theorem 7)

$$
\begin{gathered}
\left(I_{b-}^{\alpha} D_{b-}^{\alpha} f\right)(x)=(-1)^{m}\left(I_{b-}^{\alpha} I_{b-}^{m-\alpha} f^{(m)}\right)(x)= \\
(-1)^{m}\left(I_{b-}^{\alpha+m-\alpha} f^{(m)}\right)(x)=(-1)^{m}\left(I_{b-}^{m} f^{(m)}\right)(x),
\end{gathered}
$$

almost everywhere on $[a, b]$.

I.e.

$$
\left(I_{b-}^{\alpha} D_{b-}^{\alpha} f\right)(x)=(-1)^{m}\left(I_{b-}^{m} f^{(m)}\right)(x),
$$

for almost all $x \in[a, b]$.

Notice here that

$$
\left(I_{b-}^{m} f^{(m)}\right)(x)=\frac{1}{(m-1) !} \int_{x}^{b}(z-x)^{m-1} f^{(m)}(z) d z \in L_{1}([a, b], X)
$$

and exists for almost all $x \in[a, b]$, by Theorem 3 .

We have proved, by (2.42), that

$$
\frac{1}{\Gamma(\alpha)} \int_{x}^{b}(z-x)^{\alpha-1}\left(D_{b-}^{\alpha} f\right)(z) d z=\frac{1}{(m-1) !} \int_{b}^{x}(x-z)^{m-1} f^{(m)}(z) d z,
$$

for almost all $x \in[a, b]$. 
We present the following right fractional Taylor's formula

Theorem 15. Let $[a, b] \subset \mathbf{R}, X$ be a Banach space, $\alpha>0, m=\lceil\alpha\rceil$, $f \in C^{m-1}([a, b], X)$. Set

$$
F_{x}(t):=\sum_{i=0}^{m-1} \frac{(x-t)^{i}}{i !} f^{(i)}(t), \quad \forall t \in[x, b],
$$

where $x \in[a, b]$.

Assume that $f^{(m)}$ exists outside a $\mu$-null Borel set $B_{x} \subseteq[x, b]$, such that

$$
h_{1}\left(F_{x}\left(B_{x}\right)\right)=0 \text {, where } x \in[a, b] .
$$

We also assume that $f^{(m)} \in L_{1}([a, b], X)$. Then

$$
f(x)=\sum_{i=0}^{m-1} \frac{(x-b)^{i}}{i !} f^{(i)}(b)+\frac{1}{\Gamma(\alpha)} \int_{x}^{b}(z-x)^{\alpha-1}\left(D_{b-}^{\alpha} f\right)(z) d z,
$$

for $x \in[a, b]$.

Proof. We use Theorem 12.

Clearly it holds $\left(f(\cdot)-\sum_{i=0}^{m-1} \frac{(\cdot-b)^{i}}{i !} f^{(i)}(b)\right) \in C([a, b], X)$, that is (by (35) $\frac{1}{(m-1) !} \int_{b}^{x}(x-t)^{m-1} f^{(m)}(t) d t \in C([a, b], X)$ as a function of $x \in$ $[a, b]$. Hence (44) holds as an equality over $[a, b]$ (by Tonelli's theorem), therefore $\frac{1}{\Gamma(\alpha)} \int_{x}^{b}(z-x)^{\alpha-1}\left(D_{b-}^{\alpha} f\right)(z) d z \in C([a, b], X)$, as a function of $x \in[a, b]$. Now (2.47) is valid.

More generally we get

Theorem 16. Let $[a, b] \subset \mathbf{R}, X$ be a Banach space, $\alpha>0, m=\lceil\alpha\rceil$, $f \in C^{m-1}([a, b], X)$. Set

$$
F_{x}(t):=\sum_{i=0}^{m-1} \frac{(x-t)^{i}}{i !} f^{(i)}(t), \forall t \in[x, b],
$$

where $x \in[a, b]$.

Assume that $f^{(m)}$ exists outside a $\mu$-null Borel set $B_{x} \subseteq[x, b]$, such that 


$$
h_{1}\left(F_{x}\left(B_{x}\right)\right)=0, \forall x \in[a, b] .
$$

We also assume that $f^{(m)} \in L_{1}([a, b], X)$. Then

$$
f(x)=\sum_{i=0}^{m-1} \frac{(x-b)^{i}}{i !} f^{(i)}(b)+\frac{1}{\Gamma(\alpha)} \int_{x}^{b}(z-x)^{\alpha-1}\left(D_{b-}^{\alpha} f\right)(z) d z,
$$

$\forall x \in[a, b]$.

Proof. By Theorem 15 .

Remark 17. (to Theorem 16) By (2.50), we have

$$
\left(I_{b-}^{\alpha} D_{b-}^{\alpha} f\right)(x)=\frac{1}{\Gamma(\alpha)} \int_{x}^{b}(z-x)^{\alpha-1}\left(D_{b-}^{\alpha} f\right)(z) d z \in C([a, b], X)
$$

as a function of $x \in[a, b]$.

We have also

Corollary 18. (to Theorem 16) All as in Theorem 16. Assume that $f^{(i)}(b)=$ $0, i=0,1, \ldots, m-1$. Then

$$
f(x)=\frac{1}{\Gamma(\alpha)} \int_{x}^{b}(z-x)^{\alpha-1}\left(D_{b-}^{\alpha} f\right)(z) d z,
$$

$\forall x \in[a, b]$.

Next we present Ostrowski type inequalities at right fractional level for Banach valued functions. See also [3].

Theorem 19. Let $\alpha>0, m=\lceil\alpha\rceil$. Here all as in Theorem t16. Assume $f^{(k)}(b)=0, k=1, \ldots, m-1$, and $D_{b-}^{\alpha} f \in L_{\infty}([a, b], X)$. Then

$$
\left\|\frac{1}{b-a} \int_{a}^{b} f(x) d x-f(b)\right\| \leq \frac{\left\|D_{b-}^{\alpha} f\right\|_{L_{\infty}([a, b], X)}}{\Gamma(\alpha+2)}(b-a)^{\alpha} .
$$


Proof. Let $x \in[a, b]$. We have by (2.50) that

$$
f(x)-f(b)=\frac{1}{\Gamma(\alpha)} \int_{x}^{b}(J-x)^{\alpha-1} D_{b-}^{\alpha} f(J) d J .
$$

Thus

$$
\begin{gathered}
\|f(x)-f(b)\|=\frac{1}{\Gamma(\alpha)} \int_{x}^{b}(J-x)^{\alpha-1}\left\|D_{b-}^{\alpha} f(J)\right\| d J \\
\leq \frac{1}{\Gamma(\alpha)}\left(\int_{x}^{b}(J-x)^{\alpha-1} d J\right)\left\|D_{b-}^{\alpha} f\right\|_{L_{\infty}([a, b], X)}= \\
\frac{1}{\Gamma(\alpha)}\left(\left.\frac{(J-x)^{\alpha}}{\alpha}\right|_{x} ^{b}\right)\left\|D_{b-}^{\alpha} f\right\|_{L_{\infty}([a, b], X)}=\frac{1}{\Gamma(\alpha+1)}(b-x)^{\alpha}\left\|D_{b-}^{\alpha} f\right\|_{L_{\infty}([a, b], X)} .
\end{gathered}
$$

Therefore

$$
\|f(x)-f(b)\| \leq \frac{(b-x)^{\alpha}}{\Gamma(\alpha+1)}\left\|D_{b-}^{\alpha} f\right\|_{L_{\infty}([a, b], X)}, \quad \forall x \in[a, b] .
$$

Hence it holds

$$
\begin{gathered}
\left\|\frac{1}{b-a} \int_{a}^{b} f(x) d x-f(b)\right\|=\left\|\frac{1}{b-a} \int_{a}^{b}(f(x)-f(b)) d x\right\| \leq \\
\frac{1}{b-a} \int_{a}^{b}\|f(x)-f(b)\| d x \leq \frac{1}{(b-a)} \int_{a}^{b} \frac{(b-x)^{\alpha}}{\Gamma(\alpha+1)}\left\|D_{b-}^{\alpha} f\right\|_{L_{\infty}([a, b], X)} d x= \\
\frac{\left\|D_{b-}^{\alpha} f\right\|_{L_{\infty}([a, b], X)}}{(b-a) \Gamma(\alpha+1)} \int_{a}^{b}(b-x)^{\alpha} d x=\frac{\left\|D_{b-}^{\alpha} f\right\|_{L_{\infty}([a, b], X)}}{(b-a) \Gamma(\alpha+1)}\left(-\left(\left.\frac{(b-x)^{\alpha+1}}{\alpha+1}\right|_{a} ^{b}\right)\right) \\
=\frac{\left\|D_{b-}^{\alpha} f\right\|_{L_{\infty}([a, b], X)}}{(2.56)}(-1)\left(0-\frac{(b-a)^{\alpha+1}}{\alpha(\alpha+1)}\right)= \\
\frac{\left\|D_{b-}^{\alpha} f\right\|_{L_{\infty}([a, b], X)}(b-a)^{\alpha+1}}{(b-a) \Gamma(\alpha+2)} \frac{\left\|D_{b-}^{\alpha} f\right\|_{L_{\infty}([a, b], X)}(b-a)^{\alpha}}{\Gamma(\alpha+2)},
\end{gathered}
$$


proving the claim.

We also give

Theorem 20. Let $\alpha \geq 1, m=\lceil\alpha\rceil$. Here all as in Theorem 16. Assume that $f^{(k)}(b)=0, k=1, \ldots, m-1$, and $D_{b-}^{\alpha} f \in L_{1}([a, b], X)$. Then

$$
\left\|\frac{1}{b-a} \int_{a}^{b} f(x) d x-f(b)\right\| \leq \frac{\left\|D_{b-}^{\alpha} f\right\|_{L_{1}([a, b], X)}}{\Gamma(\alpha+1)}(b-a)^{\alpha-1}
$$

Proof. We have again

$$
\begin{aligned}
\| f(x)- & f(b)\left\|\leq \frac{1}{\Gamma(\alpha)} \int_{x}^{b}(J-x)^{\alpha-1}\right\| D_{b-}^{\alpha} f(J) \| d J \\
& \leq \frac{1}{\Gamma(\alpha)}(b-x)^{\alpha-1} \int_{x}^{b}\left\|D_{b-}^{\alpha} f(J)\right\| d J \\
& \leq \frac{1}{\Gamma(\alpha)}(b-x)^{\alpha-1}\left\|D_{b-}^{\alpha} f\right\|_{L_{1}([a, b], X)} .
\end{aligned}
$$

Hence

$$
\|f(x)-f(b)\| \leq \frac{\left\|D_{b-}^{\alpha} f\right\|_{L_{1}([a, b], X)}}{\Gamma(\alpha)}(b-x)^{\alpha-1}, \quad \forall x \in[a, b] .
$$

Therefore

$$
\begin{gathered}
\left\|\frac{1}{b-a} \int_{a}^{b} f(x) d x-f(b)\right\| \leq \frac{1}{b-a} \int_{a}^{b}\|f(x)-f(b)\| d x \\
\leq \frac{1}{(b-a)} \int_{a}^{b} \frac{\left\|D_{b-}^{\alpha} f\right\|_{L_{1}([a, b], X)}}{\Gamma(\alpha)}(b-x)^{\alpha-1} d x= \\
\frac{\left\|D_{b-}^{\alpha} f\right\|_{L_{1}([a, b], X)}}{(b-a) \Gamma(\alpha)} \int_{a}^{b}(b-x)^{\alpha-1} d x=\frac{\left\|D_{b-}^{\alpha} f\right\|_{L_{1}([a, b], X)}}{(b-a) \Gamma(\alpha)} \frac{(b-a)^{\alpha}}{\alpha} \\
=\frac{\left\|D_{b-}^{\alpha} f\right\|_{L_{1}([a, b], X)}}{\Gamma(\alpha+1)}(b-a)^{\alpha-1},
\end{gathered}
$$

proving the claim.

We continue with 
Theorem 21. Let $p, q>1: \frac{1}{p}+\frac{1}{q}=1, \alpha>1-\frac{1}{p}, m=\lceil\alpha\rceil$. Here all as in Theorem 16. Assume that $f^{(k)}(b)=0, k=1, \ldots, m-1$, and $D_{b-}^{\alpha} f \in L_{q}([a, b], X)$. Then

$\left\|\frac{1}{b-a} \int_{a}^{b} f(x) d x-f(b)\right\| \leq \frac{\left\|D_{b-}^{\alpha} f\right\|_{L_{q}([a, b], X)}}{\Gamma(\alpha)(p(\alpha-1)+1)^{\frac{1}{p}}\left(\alpha+\frac{1}{p}\right)}(b-a)^{\alpha-1+\frac{1}{p}}$.

Proof. We have again

$$
\begin{gathered}
\|f(x)-f(b)\| \leq \frac{1}{\Gamma(\alpha)} \int_{x}^{b}(J-x)^{\alpha-1}\left\|D_{b-}^{\alpha} f(J)\right\| d J \\
\leq \frac{1}{\Gamma(\alpha)}\left(\int_{x}^{b}(J-x)^{p(\alpha-1)} d J\right)^{\frac{1}{p}}\left(\int_{x}^{b}\left\|D_{b-}^{\alpha} f(J)\right\|^{q} d J\right)^{\frac{1}{q}} \\
\leq \frac{1}{\Gamma(\alpha)} \frac{(b-x)^{(\alpha-1)+\frac{1}{p}}}{(p(\alpha-1)+1)^{\frac{1}{p}}}\left(\int_{x}^{b}\left\|D_{b-}^{\alpha} f(J)\right\|^{q} d J\right)^{\frac{1}{q}} \\
\leq \frac{1}{\Gamma(\alpha)} \frac{(b-x)^{(\alpha-1)+\frac{1}{p}}}{(p(\alpha-1)+1)^{\frac{1}{p}}}\left\|D_{b-}^{\alpha} f\right\|_{L_{q}([a, b], X)} \cdot
\end{gathered}
$$

Therefore

$$
\|f(x)-f(b)\| \leq \frac{\left\|D_{b-}^{\alpha} f\right\|_{L_{q}([a, b], X)}}{\Gamma(\alpha)(p(\alpha-1)+1)^{\frac{1}{p}}}(b-x)^{\alpha-1+\frac{1}{p}}, \quad \forall x \in[a, b] .
$$

Hence

$$
\left\|\frac{1}{b-a} \int_{a}^{b} f(x) d x-f(b)\right\| \leq \frac{1}{b-a} \int_{a}^{b}\|f(x)-f(b)\| d x
$$


Strong right fractional calculus for Banach space valued functions 167

$$
\begin{gathered}
\leq \frac{\left\|D_{b-}^{\alpha} f\right\|_{L_{q}([a, b], X)}}{(b-a) \Gamma(\alpha)(p(\alpha-1)+1)^{\frac{1}{p}}} \int_{a}^{b}(b-x)^{\alpha-1+\frac{1}{p}} d x= \\
\frac{\left\|D_{b-}^{\alpha} f\right\|_{L_{q}([a, b], X)}}{\Gamma(\alpha)(p(\alpha-1)+1)^{\frac{1}{p}}} \frac{(b-a)^{\alpha-1+\frac{1}{p}}}{\left(\alpha+\frac{1}{p}\right)} .
\end{gathered}
$$

Corollary 22. Let $\alpha>\frac{1}{2}, m=\lceil\alpha\rceil$. All as in Theorem 16. Assume $f^{(k)}(b)=0, k=1, \ldots, m-1, D_{b-}^{\alpha} f \in L_{2}([a, b], X)$. Then

$$
\left\|\frac{1}{b-a} \int_{a}^{b} f(x) d x-f(b)\right\| \leq \frac{\left\|D_{b-}^{\alpha} f\right\|_{L_{2}([a, b], X)}}{\Gamma(\alpha)(\sqrt{2 \alpha-1})\left(\alpha+\frac{1}{2}\right)}(b-a)^{\alpha-\frac{1}{2}} .
$$

We give

Proposition 23. Inequality (2.53) is sharp; namely it is attained by

$$
f(x)=(b-x)^{\alpha} \vec{i}, \alpha>0, \alpha \notin \mathbf{N}, x \in[a, b],
$$

$$
\vec{i} \in X \text {, such that }\|\vec{i}\|=1 \text {. }
$$

Proof. (see also [4], pp. 26-27) We see that

$$
\begin{gathered}
\mathrm{f}^{\prime}(x)=-\alpha(b-x)^{\alpha-1} \vec{i}, f^{\prime \prime}(x)=(-1)^{2} \alpha(\alpha-1)(b-x)^{\alpha-2} \vec{i}, \ldots, \\
f^{(m-1)}(x)=(-1)^{m-1} \alpha(\alpha-1)(\alpha-2) \ldots(\alpha-m+2)(b-x)^{\alpha-m+1} \vec{i},
\end{gathered}
$$

and

$$
\mathrm{f}^{(m)}(x)=(-1)^{m} \alpha(\alpha-1)(\alpha-2) \ldots(\alpha-m+2)(\alpha-m+1)(b-x)^{\alpha-m} \vec{i} .
$$


Here $f^{(m)}$ is continuous on $[a, b)$, and $f^{(m)} \in L_{1}([a, b], X)$. All assumptions of Theorem 16 are easily fulfilled.

Thus

$$
\begin{gathered}
D_{b-}^{\alpha} f(x)=\frac{\vec{i}(-1)^{2 m}}{\Gamma(m-\alpha)} \alpha(\alpha-1) \ldots(\alpha-m+1) \int_{x}^{b}(J-x)^{m-\alpha-1}(b-J)^{\alpha-m} d J \\
=\frac{\vec{i} \alpha(\alpha-1) \ldots(\alpha-m+1)}{\Gamma(m-\alpha)} \int_{x}^{b}(b-J)^{(\alpha-m+1)-1}(J-x)^{(m-\alpha)-1} d J
\end{gathered}
$$

$$
\begin{aligned}
& =\frac{\vec{i} \alpha(\alpha-1) \ldots(\alpha-m+1)}{\Gamma(m-\alpha)} \frac{\Gamma(\alpha-m+1) \Gamma(m-\alpha)}{\Gamma(1)} \\
= & \vec{i} \alpha(\alpha-1) \ldots(\alpha-m+1) \Gamma(\alpha-m+1)=\Gamma(\alpha+1) \vec{i} .
\end{aligned}
$$

That is

$$
D_{b-}^{\alpha} f(x)=\Gamma(\alpha+1) \vec{i}, \forall x \in[a, b] .
$$

Also we see that $f^{(k)}(b)=0, k=0,1, \ldots, m-1$, and $D_{b-}^{\alpha} f \in L_{\infty}([a, b], X)$. So $f$ fulfills all assumptions of Theorem 19. Next we see

$$
\text { R.H.S. }(2.53)=\frac{\Gamma(\alpha+1)}{\Gamma(\alpha+2)}(b-a)^{\alpha}=\frac{(b-a)^{\alpha}}{(\alpha+1)} \text {. }
$$

L.H.S. $(2.53)=\frac{1}{b-a} \int_{a}^{b}(b-x)^{\alpha} d x=\frac{1}{b-a} \frac{(b-a)^{\alpha+1}}{(\alpha+1)}=\frac{(b-a)^{\alpha}}{(\alpha+1)}$,

proving attainability and sharpness of (2.53).

We continue with a Poincaré like right fractional inequality: 
Theorem 24. Let $p, q>1: \frac{1}{p}+\frac{1}{q}=1$, and $\alpha>\frac{1}{q}, m=\lceil\alpha\rceil$. Here all as in Theorem 16.

Assume that $f^{(k)}(b)=0, k=0,1, \ldots, m-1$, and $D_{b-}^{\alpha} f \in L_{q}([a, b], X)$, where $X$ is a Banach space. Then

$$
\|f\|_{L_{q}([a, b], X)} \leq \frac{(b-a)^{\alpha}\left\|D_{b-}^{\alpha} f\right\|_{L_{q}([a, b], X)}}{\Gamma(\alpha)(p(\alpha-1)+1)^{\frac{1}{p}}(q \alpha)^{\frac{1}{q}}} .
$$

Proof. We have that (by (2.52))

$$
f(x)=\frac{1}{\Gamma(\alpha)} \int_{x}^{b}(z-x)^{\alpha-1}\left(D_{b-}^{\alpha} f\right)(z) d z, \quad \forall x \in[a, b] .
$$

Hence

$$
\begin{gathered}
\|f(x)\|=\frac{1}{\Gamma(\alpha)}\left\|\int_{x}^{b}(z-x)^{\alpha-1}\left(D_{b-}^{\alpha} f\right)(z) d z\right\| \leq \\
\frac{1}{\Gamma(\alpha)} \int_{x}^{b}(z-x)^{\alpha-1}\left\|D_{b-}^{\alpha} f(z)\right\| d z \leq \\
\frac{1}{\Gamma(\alpha)}\left(\int_{x}^{b}(z-x)^{p(\alpha-1)} d z\right)^{\frac{1}{p}}\left(\int_{x}^{b}\left\|\left(D_{b-}^{\alpha} f\right)(z)\right\|^{q} d z\right)^{\frac{1}{q}} \leq \\
\frac{1}{\Gamma(\alpha)} \frac{(b-x)^{\frac{p(\alpha-1)+1}{p}}}{(p(\alpha-1)+1)^{\frac{1}{p}}}\left\|D_{b-}^{\alpha} f\right\|_{L_{q}([a, b], X)} .
\end{gathered}
$$

We have proved that

$$
\|f(x)\| \leq \frac{1}{\Gamma(\alpha)} \frac{(b-x)^{\frac{p(\alpha-1)+1}{p}}}{(p(\alpha-1)+1)^{\frac{1}{p}}}\left\|D_{b-}^{\alpha} f\right\|_{L_{q}([a, b], X)}, \quad \forall x \in[a, b] .
$$

Then

$$
\|f(x)\|^{q} \leq \frac{(b-x)^{(p(\alpha-1)+1) \frac{q}{p}}}{(\Gamma(\alpha))^{q}(p(\alpha-1)+1)^{\frac{q}{p}}}\left\|D_{b-}^{\alpha} f\right\|_{L_{q}([a, b], X)}^{q}
$$


$\forall x \in[a, b]$.

Hence it holds

$$
\int_{a}^{b}\|f(x)\|^{q} d x \leq \frac{(b-a)^{q \alpha}}{(\Gamma(\alpha))^{q}(p(\alpha-1)+1)^{\frac{q}{p}} q \alpha}\left\|D_{b-}^{\alpha} f\right\|_{L_{q}([a, b], X)}^{q} .
$$

The last inequality implies

$$
\left(\int_{a}^{b}\|f(x)\|^{q} d x\right)^{\frac{1}{q}} \leq \frac{(b-a)^{\alpha}\left\|D_{b-}^{\alpha} f\right\|_{L_{q}([a, b], X)}}{\Gamma(\alpha)(p(\alpha-1)+1)^{\frac{1}{p}}(q \alpha)^{\frac{1}{q}}}
$$

proving the claim.

Next comes a right Sobolev like fractional inequality:

Theorem 25. All as in the last Theorem 24. Let $r>0$. Then

$$
\|f\|_{L_{r}([a, b], X)} \leq \frac{(b-a)^{\alpha-\frac{1}{q}+\frac{1}{r}}\left\|D_{b-}^{\alpha} f\right\|_{L_{q}([a, b], X)}}{\Gamma(\alpha)(p(\alpha-1)+1)^{\frac{1}{p}}\left(r\left(\alpha-\frac{1}{q}\right)+1\right)^{\frac{1}{r}}} .
$$

Proof. As in the last theorem's proof we get that

$$
\|f(x)\| \leq \frac{1}{\Gamma(\alpha)} \frac{(b-x)^{\alpha-\frac{1}{q}}}{(p(\alpha-1)+1)^{\frac{1}{p}}}\left\|D_{b-}^{\alpha} f\right\|_{L_{q}([a, b], X)}, \quad \forall x \in[a, b] .
$$

Since $r>0$, we get

$$
\|f(x)\|^{r} \leq \frac{1}{(\Gamma(\alpha))^{r}} \frac{(b-x)^{r\left(\alpha-\frac{1}{q}\right)}}{(p(\alpha-1)+1)^{\frac{r}{p}}}\left\|D_{b-}^{\alpha} f\right\|_{L_{q}([a, b], X)}^{r}, \quad \forall x \in[a, b] .
$$

Hence it holds 
$\int_{a}^{b}\|f(x)\|^{r} d x \leq \frac{(b-a)^{r\left(\alpha-\frac{1}{q}\right)+1}}{(\Gamma(\alpha))^{r}(p(\alpha-1)+1)^{\frac{r}{p}}\left(r\left(\alpha-\frac{1}{q}\right)+1\right)}\left\|D_{b-}^{\alpha} f\right\|_{L_{q}([a, b], X)}^{r}$.

That is

$$
\left(\int_{a}^{b}\|f(x)\|^{r} d x\right)^{\frac{1}{r}} \leq \frac{(b-a)^{\alpha-\frac{1}{q}+\frac{1}{r}}}{\Gamma(\alpha)(p(\alpha-1)+1)^{\frac{1}{p}}\left(r\left(\alpha-\frac{1}{q}\right)+1\right)^{\frac{1}{r}}}\left\|D_{b-}^{\alpha} f\right\|_{L_{q}([a, b], X)},
$$

proving the claim.

We give the following Opial type right fractional inequality:

Theorem 26. Let $p, q>1: \frac{1}{p}+\frac{1}{q}=1$, and $\alpha>\frac{1}{q}, m:=\lceil\alpha\rceil$. Let $[a, b] \subset \mathbf{R}, X$ a Banach space, and $f \in C^{m-1}([a, b], X)$. Set

$$
F_{x}(t):=\sum_{i=0}^{m-1} \frac{(x-t)^{i}}{i !} f^{(i)}(t), \forall t \in[x, b], \text { where } x \in[a, b] .
$$

Assume that $f^{(m)}$ exists outside a $\mu$-null Borel set $B_{x} \subseteq[x, b]$, such that

$$
h_{1}\left(F_{x}\left(B_{x}\right)\right)=0, \forall x \in[a, b] .
$$

We assume that $f^{(m)} \in L_{\infty}([a, b], X)$. Assume also that $f^{(k)}(b)=0$, $k=0,1, \ldots, m-1$. Then

$$
\begin{gathered}
\int_{x}^{b}\|f(w)\|\left\|\left(D_{b-}^{\alpha} f\right)(w)\right\| d w \leq \\
\frac{(b-x)^{\alpha-1+\frac{2}{p}}}{2^{\frac{1}{q}} \Gamma(\alpha)((p(\alpha-1)+1)(p(\alpha-1)+2))^{\frac{1}{p}}}\left(\int_{x}^{b}\left\|\left(D_{b-}^{\alpha} f\right)(z)\right\|^{q} d z\right)^{\frac{2}{q}},
\end{gathered}
$$

$\forall x \in[a, b]$. 
Proof. By (2.52) we get

$$
f(x)=\frac{1}{\Gamma(\alpha)} \int_{x}^{b}(z-x)^{\alpha-1}\left(D_{b-}^{\alpha} f\right)(z) d z, \quad \forall x \in[a, b] .
$$

Let $x \leq w \leq b$, then we have

$$
f(w)=\frac{1}{\Gamma(\alpha)} \int_{w}^{b}(z-w)^{\alpha-1}\left(D_{b-}^{\alpha} f\right)(z) d z .
$$

Furthermore it holds

$$
\begin{gathered}
\|f(w)\| \leq \frac{1}{\Gamma(\alpha)} \int_{w}^{b}(z-w)^{\alpha-1}\left\|\left(D_{b-}^{\alpha} f\right)(z)\right\| d z \leq \\
\frac{1}{\Gamma(\alpha)}\left(\int_{w}^{b}(z-w)^{p(\alpha-1)} d z\right)^{\frac{1}{p}}\left(\int_{w}^{b}\left\|\left(D_{b-}^{\alpha} f\right)(z)\right\|^{q} d z\right)^{\frac{1}{q}}= \\
\frac{1}{\Gamma(\alpha)} \frac{(b-w)^{\frac{(p(\alpha-1)+1)}{p}}}{(p(\alpha-1)+1)^{\frac{1}{p}}}\left(\int_{w}^{b}\left\|\left(D_{b-}^{\alpha} f\right)(z)\right\|^{q} d z\right)^{\frac{1}{q}}= \\
\frac{1}{\Gamma(\alpha)} \frac{(b-w)^{\alpha-\frac{1}{q}}}{(p(\alpha-1)+1)^{\frac{1}{p}}}(z(w))^{\frac{1}{q}}
\end{gathered}
$$

where

$$
z(w):=\int_{w}^{b}\left\|\left(D_{b-}^{\alpha} f\right)(z)\right\|^{q} d z
$$

all $x \leq w \leq b, z(b)=0$.

Thus

$$
-z(w):=\int_{b}^{w}\left\|\left(D_{b-}^{\alpha} f\right)(z)\right\|^{q} d z
$$

and

$$
(-z(w))^{\prime}=\left\|\left(D_{b-}^{\alpha} f\right)(w)\right\|^{q} \geq 0
$$

and

$$
\left\|\left(D_{b-}^{\alpha} f\right)(w)\right\|=\left((-z(w))^{\prime}\right)^{\frac{1}{q}}=\left(-(z(w))^{\prime}\right)^{\frac{1}{q}} .
$$

Therefore we obtain 


$$
\|f(w)\|\left\|\left(D_{b-}^{\alpha} f\right)(w)\right\| \leq \frac{(b-w)^{\alpha-\frac{1}{q}}}{\Gamma(\alpha)(p(\alpha-1)+1)^{\frac{1}{p}}}\left(z(w)\left(-(z(w))^{\prime}\right)\right)^{\frac{1}{q}}
$$

all $x \leq w \leq b$.

Integrating (2.94) we get

$$
\begin{gathered}
\int_{x}^{b}\|f(w)\|\left\|\left(D_{b-}^{\alpha} f\right)(w)\right\| d w \leq \\
\frac{1}{\Gamma(\alpha)(p(\alpha-1)+1)^{\frac{1}{p}}} \int_{x}^{b}(b-w)^{\alpha-\frac{1}{q}}\left(z(w)\left(-z^{\prime}(w)\right)\right)^{\frac{1}{q}} d w \leq
\end{gathered}
$$

$\frac{1}{\Gamma(\alpha)(p(\alpha-1)+1)^{\frac{1}{p}}}\left(\int_{x}^{b}(b-w)^{\left(\alpha-\frac{1}{q}\right) p} d w\right)^{\frac{1}{p}}\left(\int_{x}^{b}\left(z(w)\left(-z^{\prime}(w)\right)\right) d w\right)^{\frac{1}{q}}=$ $(2.95)$

$$
\begin{gathered}
\frac{1}{\Gamma(\alpha)(p(\alpha-1)+1)^{\frac{1}{p}}} \frac{(b-x)^{\alpha-1+\frac{2}{p}}}{(p(\alpha-1)+2)^{\frac{1}{p}}} \frac{(z(x))^{\frac{2}{q}}}{2^{\frac{1}{q}}}= \\
\frac{(b-x)^{\alpha-1+\frac{2}{p}}}{2^{\frac{1}{q}} \Gamma(\alpha)[(p(\alpha-1)+1)(p(\alpha-1)+2)]^{\frac{1}{p}}}\left(\int_{x}^{b}\left\|\left(D_{b-}^{\alpha} f\right)(z)\right\|^{q} d z\right)^{\frac{2}{q}},
\end{gathered}
$$

$\forall x \in[a, b]$, proving the claim.

Next we present a Hilbert-Pachpatte right fractional inequality: 
Theorem 27. Let $p, q>1: \frac{1}{p}+\frac{1}{q}=1$, and $\alpha_{1}>\frac{1}{q}, \alpha_{2}>\frac{1}{p}, m_{i}:=\left\lceil\alpha_{i}\right\rceil$, $i=1,2$. Here $\left[a_{i}, b_{i}\right] \subset \mathbf{R}, i=1,2 ; X$ is a Banach space. Let $f_{i} \in$ $C^{m_{i}-1}\left(\left[a_{i}, b_{i}\right], X\right), i=1,2$. Set

$$
F_{x_{i}}\left(t_{i}\right):=\sum_{j_{i}=0}^{m_{i}-1} \frac{\left(x_{i}-t_{i}\right)^{j_{i}}}{j_{i} !} f_{i}^{\left(j_{i}\right)}\left(t_{i}\right),
$$

$\forall t_{i} \in\left[x_{i}, b_{i}\right]$, where $x_{i} \in\left[a_{i}, b_{i}\right] ; i=1,2$. Assume that $f_{i}^{\left(m_{i}\right)}$ exists outside a $\mu$-null Borel set $B_{x_{i}} \subseteq\left[x_{i}, b_{i}\right]$, such that

$$
h_{1}\left(F_{x_{i}}\left(B_{x_{i}}\right)\right)=0, \quad \forall x_{i} \in\left[a_{i}, b_{i}\right] ; i=1,2 .
$$

We also assume that $f_{i}^{\left(m_{i}\right)} \in L_{1}\left(\left[a_{i}, b_{i}\right], X\right)$, and

$$
f_{i}^{\left(k_{i}\right)}\left(b_{i}\right)=0, \quad k_{i}=0,1, \ldots, m_{i}-1 ; i=1,2,
$$

and

$$
\left(D_{b_{1}-}^{a_{1}} f_{1}\right) \in L_{q}\left(\left[a_{1}, b_{1}\right], X\right), \quad\left(D_{b_{2}-}^{\alpha_{2}} f_{2}\right) \in L_{p}\left(\left[a_{2}, b_{2}\right], X\right)
$$

Then

$$
\begin{gathered}
\int_{a_{1}}^{b_{1}} \int_{a_{2}}^{b_{2}} \frac{\left\|f_{1}\left(x_{1}\right)\right\|\left\|f_{2}\left(x_{2}\right)\right\| d x_{1} d x_{2}}{\left(\frac{\left(b_{1}-x_{1}\right)^{p\left(\alpha_{1}-1\right)+1}}{p\left(p\left(\alpha_{1}-1\right)+1\right)}+\frac{\left(b_{2}-x_{2}\right)^{q\left(\alpha_{2}-1\right)+1}}{q\left(q\left(\alpha_{2}-1\right)+1\right)}\right)} \leq \\
\frac{\left(b_{1}-a_{1}\right)\left(b_{2}-a_{2}\right)}{\Gamma\left(\alpha_{1}\right) \Gamma\left(\alpha_{2}\right)}\left\|D_{b_{1}-}^{\alpha_{1}} f_{1}\right\|_{L_{q}\left(\left[a_{1}, b_{1}\right], X\right)}\left\|D_{b_{2}-}^{\alpha_{2}} f_{2}\right\|_{L_{p}\left(\left[a_{2}, b_{2}\right], X\right)}
\end{gathered}
$$

Proof. We have that (by $(2.52)$ )

$f_{i}\left(x_{i}\right)=\frac{1}{\Gamma\left(\alpha_{i}\right)} \int_{x_{i}}^{b_{i}}\left(z_{i}-x_{i}\right)^{\alpha_{i}-1}\left(D_{b_{i}-}^{\alpha_{i}} f_{i}\right)\left(z_{i}\right) d z_{i}, \quad \forall x_{i} \in\left[a_{i}, b_{i}\right], i=1,2$. 
Then

$$
\left\|f_{i}\left(x_{i}\right)\right\| \leq \frac{1}{\Gamma\left(\alpha_{i}\right)} \int_{x_{i}}^{b_{i}}\left(z_{i}-x_{i}\right)^{\alpha_{i}-1}\left\|\left(D_{b_{i}-}^{\alpha_{i}} f_{i}\right)\left(z_{i}\right)\right\| d z_{i},
$$

$\forall x_{i} \in\left[a_{i}, b_{i}\right], i=1,2$.

We get as before,

$$
\left\|f_{1}\left(x_{1}\right)\right\| \leq \frac{1}{\Gamma\left(\alpha_{1}\right)} \frac{\left(b_{1}-x_{1}\right)^{\frac{p\left(\alpha_{1}-1\right)+1}{p}}}{\left(p\left(\alpha_{1}-1\right)+1\right)^{\frac{1}{p}}}\left\|D_{b_{1}-}^{\alpha_{1}} f_{1}\right\|_{L_{q}\left(\left[a_{1}, b_{1}\right], X\right)}
$$

and

$$
\left\|f_{2}\left(x_{2}\right)\right\| \leq \frac{1}{\Gamma\left(\alpha_{2}\right)} \frac{\left(b_{2}-x_{2}\right)^{\frac{q\left(\alpha_{2}-1\right)+1}{q}}}{\left(q\left(\alpha_{2}-1\right)+1\right)^{\frac{1}{q}}}\left\|D_{b_{2}-}^{\alpha_{2}} f_{2}\right\|_{L_{p}\left(\left[a_{2}, b_{2}\right], X\right)} .
$$

Hence we have

$$
\begin{gathered}
\left\|f_{1}\left(x_{1}\right)\right\|\left\|f_{2}\left(x_{2}\right)\right\| \leq \frac{1}{\Gamma\left(\alpha_{1}\right) \Gamma\left(\alpha_{2}\right)\left(p\left(\alpha_{1}-1\right)+1\right)^{\frac{1}{p}}\left(q\left(\alpha_{2}-1\right)+1\right)^{\frac{1}{q}}} . \\
\left(b_{1}-x_{1}\right)^{\frac{p\left(\alpha_{1}-1\right)+1}{p}}\left(b_{2}-x_{2}\right)^{\frac{q\left(\alpha_{2}-1\right)+1}{q}}\left\|D_{b_{1}-}^{\alpha_{1}} f_{1}\right\|_{L_{q}\left(\left[a_{1}, b_{1}\right], X\right)}\left\|D_{b_{2}-}^{\alpha_{2}} f_{2}\right\|_{L_{p}\left(\left[a_{2}, b_{2}\right], X\right)}
\end{gathered}
$$

(using Young's inequality for $a, b \geq 0, a^{\frac{1}{p}} b^{\frac{1}{q}} \leq \frac{a}{p}+\frac{b}{q}$ )

$$
\begin{aligned}
& (2.107) \leq \frac{1}{\Gamma\left(\alpha_{1}\right) \Gamma\left(\alpha_{2}\right)}\left(\frac{\left(b_{1}-x_{1}\right)^{p\left(\alpha_{1}-1\right)+1}}{p\left(p\left(\alpha_{1}-1\right)+1\right)}+\frac{\left(b_{2}-x_{2}\right)^{q\left(\alpha_{2}-1\right)+1}}{q\left(q\left(\alpha_{2}-1\right)+1\right)}\right) . \\
& \quad\left\|D_{b_{1}-f_{1}}^{\alpha_{1}}\right\|_{L_{q}\left(\left[a_{1}, b_{1}\right], X\right)}\left\|D_{b_{2}-}^{\alpha_{2}} f_{2}\right\|_{L_{p}\left(\left[a_{2}, b_{2}\right], X\right)}, \\
& \forall x_{i} \in\left[a_{i}, b_{i}\right] ; i=1,2 .
\end{aligned}
$$

So far we have

$$
\begin{gathered}
\frac{\left\|f_{1}\left(x_{1}\right)\right\|\left\|f_{2}\left(x_{2}\right)\right\|}{\left(\frac{\left(b_{1}-x_{1}\right)^{p\left(\alpha_{1}-1\right)+1}}{p\left(p\left(\alpha_{1}-1\right)+1\right)}+\frac{\left(b_{2}-x_{2}\right)^{q\left(\alpha_{2}-1\right)+1}}{q\left(q\left(\alpha_{2}-1\right)+1\right)}\right)} \leq \\
\frac{\left\|D_{b_{1}-}^{\alpha_{1}} f_{1}\right\|_{L_{q}\left(\left[a_{1}, b_{1}\right], X\right)}\left\|D_{b_{2}-}^{\alpha_{2}} f_{2}\right\|_{L_{p}\left(\left[a_{2}, b_{2}\right], X\right)}}{\Gamma\left(\alpha_{1}\right) \Gamma\left(\alpha_{2}\right)}
\end{gathered}
$$


$\forall x_{i} \in\left[a_{i}, b_{i}\right] ; i=1,2$.

The denominator in (2.108) can be zero only when $x_{1}=b_{1}$ and $x_{2}=b_{2}$. Integrating (2.108) over $\left[a_{1}, b_{1}\right] \times\left[a_{2}, b_{2}\right]$ we derive inequality (2.101).

When $0<\alpha \leq 1$, Definition 13 becomes

Definition 28. Let $[a, b] \subset \mathbf{R}, X$ be a Banach space, $0<\alpha \leq 1$. We assume that $f^{\prime} \in L_{1}([a, b], X)$, where $f:[a, b] \rightarrow X$.

We define the Caputo-Bochner right fractional derivative of order $\alpha$ :

$$
\left(D_{b-}^{\alpha} f\right)(x):=-I_{b-}^{1-\alpha} f^{\prime}(x),
$$

i.e.

$$
\left(D_{b-}^{\alpha} f\right)(x)=\frac{-1}{\Gamma(1-\alpha)} \int_{x}^{b}(J-x)^{-\alpha} f^{\prime}(J) d J, \quad \forall x \in[a, b]
$$

Clearly $D_{b-}^{1} f(x)=-f^{\prime}(x)$.

Remark 29. Let $[A, B] \subset \mathbf{R}, X$ be a Banach space, $0<\alpha \leq 1, f$ : $[A, B] \rightarrow X$. We assume that $f^{(2)} \in L_{1}([A, B], X)$. Then $\lceil\alpha+1\rceil=2$, and

$$
D_{B-}^{\alpha} f^{\prime}(x)=\frac{-1}{\Gamma(1-\alpha)} \int_{x}^{B}(J-x)^{-\alpha} f^{\prime \prime}(J) d J=
$$

$$
-\frac{(-1)^{2}}{\Gamma(2-(\alpha+1)) \int_{x}^{B}(J-x)^{2-(\alpha+1)-1} f^{\prime \prime}(J) d J=-D_{B-}^{\alpha+1} f(x),}
$$

i.e.

$$
D_{B-}^{\alpha} f^{\prime}(x)=-D_{B-}^{\alpha+1} f(x),
$$

and

$$
\left\|D_{B-}^{\alpha} f^{\prime}(x)\right\|=\left\|D_{B-}^{\alpha+1} f(x)\right\|, \quad \forall x \in[A, B] .
$$

We apply Theorem 19 when $0<\alpha \leq 1$.

Theorem 30. Let $0<\alpha \leq 1,[A, B] \subset \mathbf{R}, X$ a Banach space, $f \in$ $C([A, B], X)$. Assume that $f^{\prime}$ exists outside a $\mu$-null Borel set $B_{x} \subseteq[x, B]$, such that

$$
h_{1}\left(f\left(B_{x}\right)\right)=0, \quad \forall x \in[A, B] .
$$


We assume that $f^{\prime} \in L_{1}([A, B], X)$, and $D_{B-}^{\alpha} f \in L_{\infty}([A, B], X)$. Then

$$
\left\|\frac{1}{B-A} \int_{A}^{B} f(x) d x-f(B)\right\| \leq \frac{\left\|D_{B-}^{\alpha} f\right\|_{L_{\infty}([A, B], X)}}{\Gamma(\alpha+2)}(B-A)^{\alpha} .
$$

We present the following right Caputo-Bochner fractional Landau inequality for $\|\cdot\|_{\infty}$.

Theorem 31. Let $f \in C^{1}\left(\left(-\infty, B_{0}\right], X\right)$, where $B_{0} \in \mathbf{R}$ is fixed, $0<\alpha \leq$ $1, X$ is a Banach space. For any $A, B \in\left(-\infty, B_{0}\right]: A \leq B$, we assume that $f$ fulfills: assume that $f^{\prime \prime}$ exists outside a $\mu$-null Borel set $B_{x} \subseteq[x, B]$, such that

$$
h_{1}\left(f^{\prime}\left(B_{x}\right)\right)=0, \forall x \in[A, B] .
$$

We assume that $f^{\prime \prime} \in L_{1}([A, B], X)$, and $D_{B-}^{\alpha+1} f \in L_{\infty}([A, B], X)$. We further assume that

$$
\left\|D_{B-}^{\alpha+1} f\right\|_{L_{\infty}((-\infty, B], X)} \leq\left\|D_{B_{0}-}^{\alpha+1} f\right\|_{L_{\infty}\left(\left(-\infty, B_{0}\right], X\right)}<\infty, \quad \forall B \leq B_{0} .
$$

(the last left inequality is obvious when $\alpha=1$ ), and

$$
\|f\|_{\infty,\left(-\infty, B_{0}\right]}:=t \in\left(-\infty, B_{0}\right] \sup \|f(t)\|<\infty
$$

Then

$\left\|f^{\prime}\right\|_{\infty,\left(-\infty, B_{0}\right]}:=t \in\left(-\infty, B_{0}\right] \sup \left\|f^{\prime}(t)\right\| \leq(\alpha+1)\left(\frac{2}{\alpha}\right)^{\left(\frac{\alpha}{\alpha+1}\right)}(\Gamma(\alpha+2))^{-\frac{1}{(\alpha+1)}}$

$$
\left(\|f\|_{\infty,\left(-\infty, B_{0}\right]}\right)^{\frac{\alpha}{(\alpha+1)}}\left(\left\|D_{B_{0}-}^{\alpha+1} f\right\|_{L_{\infty}\left(\left(-\infty, B_{0}\right], X\right)}\right)^{\frac{1}{(\alpha+1)}} .
$$


Proof. We have that (by Theorem 30)

$$
\left\|\frac{1}{B-A} \int_{A}^{B} f^{\prime}(x) d x-f^{\prime}(B)\right\| \leq \frac{\left\|D_{B-}^{\alpha+1} f\right\|_{L_{\infty}([A, B], X)}}{\Gamma(\alpha+2)}(B-A)^{\alpha},
$$

$\forall A, B \in\left(-\infty, B_{0}\right]: A \leq B$.

Subsequently by Theorem 10 we derive

$$
\left\|\frac{f(B)-f(A)}{B-A}-f^{\prime}(B)\right\| \leq \frac{\left\|D_{B-}^{\alpha+1} f\right\|_{L_{\infty}([A, B], X)}}{\Gamma(\alpha+2)}(B-A)^{\alpha},
$$

$\forall A, B \in\left(-\infty, B_{0}\right], A \leq B$.

Hence it holds

$$
\left\|f^{\prime}(B)\right\|-\frac{1}{B-A}\|f(B)-f(A)\| \leq \frac{\left\|D_{B-}^{\alpha+1} f\right\|_{L_{\infty}([A, B], X)}}{\Gamma(\alpha+2)}(B-A)^{\alpha},
$$

and

$$
\left\|f^{\prime}(B)\right\| \leq \frac{\|f(B)-f(A)\|}{B-A}+\frac{\left\|D_{B-}^{\alpha+1} f\right\|_{L_{\infty}([A, B], X)}}{\Gamma(\alpha+2)}(B-A)^{\alpha},
$$

$\forall A, B \in\left(-\infty, B_{0}\right]: A \leq B$.

Therefore we obtain

$$
\left\|f^{\prime}(B)\right\| \leq \frac{2\|f\|_{\infty,\left(-\infty, B_{0}\right]}}{B-A}+\frac{\left\|D_{B_{0}-}^{\alpha+1} f\right\|_{L_{\infty}\left(\left(-\infty, B_{0}\right], X\right)}}{\Gamma(\alpha+2)}(B-A)^{\alpha},
$$


$\forall A, B \in\left(-\infty, B_{0}\right]: A \leq B$.

The right hand side of (2.123) depends only on $B-A$. Consequently, it holds

$$
\left\|f^{\prime}\right\|_{\infty,\left(-\infty, B_{0}\right]} \leq \frac{2\|f\|_{\infty,\left(-\infty, B_{0}\right]}}{B-A}+\frac{\left\|D_{B_{0}-}^{\alpha+1} f\right\|_{L_{\infty}\left(\left(-\infty, B_{0}\right], X\right)}}{\Gamma(\alpha+2)}(B-A)^{\alpha} .
$$

We may call $t=B-A>0$. Thus by (2.124),

$\left\|f^{\prime}\right\|_{\infty,\left(-\infty, B_{0}\right]} \leq \frac{2\|f\|_{\infty,\left(-\infty, B_{0}\right]}}{t}+\frac{\left\|D_{B_{0}-}^{\alpha+1} f\right\|_{L_{\infty}\left(\left(-\infty, B_{0}\right], X\right)}}{\Gamma(\alpha+2)} t^{\alpha}, \quad \forall t>0$.

Set

$\mu:=2\|f\|_{\infty,\left(-\infty, B_{0}\right]}$,

$$
\theta:=\frac{\left\|D_{B_{0}-}^{\alpha+1} f\right\|_{L_{\infty}\left(\left(-\infty, B_{0}\right], X\right)}}{\Gamma(\alpha+2)},
$$

both are greater than 0 .

We consider the function

$$
y(t)=\frac{\mu}{t}+\theta t^{\alpha}, \quad 0<\alpha \leq 1, t>0 .
$$

As in [4], pp. 81-82, y has a global minimum at

$$
t_{0}=\left(\frac{\mu}{\alpha \theta}\right)^{\frac{1}{(\alpha+1)}}
$$

which is 


$$
y\left(t_{0}\right)=\left(\theta \mu^{\alpha}\right)^{\frac{1}{(\alpha+1)}}(\alpha+1) \alpha^{-\left(\frac{\alpha}{\alpha+1}\right)} .
$$

Consequently it is

$y\left(t_{0}\right)=\left(\frac{\left\|D_{B_{0}-}^{\alpha+1} f\right\|_{L_{\infty}\left(\left(-\infty, B_{0}\right], X\right)}}{\Gamma(\alpha+2)}\right)^{\frac{1}{(\alpha+1)}}\left(2\|f\|_{\infty,\left(-\infty, B_{0}\right]}\right)^{\left(\frac{\alpha}{\alpha+1}\right)}(\alpha+1) \alpha^{-\left(\frac{\alpha}{\alpha+1}\right)}$.

We have proved that

$$
\left\|f^{\prime}\right\|_{\infty,\left(-\infty, B_{0}\right]} \leq(\alpha+1)\left(\frac{2}{\alpha}\right)^{\left(\frac{\alpha}{\alpha+1}\right)}(\Gamma(\alpha+2))^{-\frac{1}{(\alpha+1)}} .
$$

$$
\left(\left\|D_{B_{0}-}^{\alpha+1} f\right\|_{L_{\infty}\left(\left(-\infty, B_{0}\right], X\right)}\right)^{\frac{1}{(\alpha+1)}}\left(\|f\|_{\infty,\left(-\infty, B_{0}\right]}\right)^{\left(\frac{\alpha}{\alpha+1}\right)}
$$

establishing the claim.

The case $B_{0}=0$ comes next

Corollary 32. (to Theorem 31) All as in Theorem 31 for $B_{0}=0$.

Then $\left\|f^{\prime}\right\|_{\infty, \mathbf{R}_{-}} \leq(\alpha+1)\left(\frac{2}{\alpha}\right)^{\left(\frac{\alpha}{\alpha+1}\right)}(\Gamma(\alpha+2))^{-\frac{1}{(\alpha+1)}}$.

$$
\left(\|f\|_{\infty, \mathbf{R}_{-}}\right)^{\left(\frac{\alpha}{\alpha+1}\right)}\left(\left\|D_{0-}^{\alpha+1} f\right\|_{L_{\infty}\left(\mathbf{R}_{-}, X\right)}\right)^{\frac{1}{(\alpha+1)}} .
$$

When $\alpha=1$ we get

Corollary 33. (to Theorem 31) Let $f \in C^{1}\left(\left(-\infty, B_{0}\right], X\right)$, where $B_{0} \in \mathbf{R}$ is fixed, $X$ is a Banach space. For any $A, B \in\left(-\infty, B_{0}\right]: A \leq B$, we assume that $f$ fulfills: assume that $f^{\prime \prime}$ exists outside a $\mu$-null Borel set $B_{x} \subseteq[x, B]$, such that

$$
h_{1}\left(f^{\prime}\left(B_{x}\right)\right)=0, \quad \forall x \in[A, B] .
$$

We assume that $f^{\prime \prime} \in L_{\infty}\left(\left(-\infty, B_{0}\right], X\right)$, and $\|f\|_{\infty,\left(-\infty, B_{0}\right]}<\infty$. Then

$$
\left\|f^{\prime}\right\|_{\infty,\left(-\infty, B_{0}\right]} \leq 2\|f\|_{\infty,\left(-\infty, B_{0}\right]}^{\frac{1}{2}}\left\|f^{\prime \prime}\right\|_{L_{\infty}\left(\left(-\infty, B_{0}\right], X\right)}^{\frac{1}{2}} .
$$

Next case of $B_{0}=0$.

Corollary 34. All as in Corollary 33. It holds

$$
\left\|f^{\prime}\right\|_{\infty, \mathbf{R}_{-}} \leq 2\|f\|_{\infty, \mathbf{R}_{-}}^{\frac{1}{2}}\left\|f^{\prime \prime}\right\|_{L_{\infty}\left(\mathbf{R}_{-}, X\right)}^{\frac{1}{2}} .
$$


See also [9].

We apply Theorem 21 when $0<\alpha \leq 1$.

Theorem 35. Let $p, q>1: \frac{1}{p}+\frac{1}{q}=1, \frac{1}{q}<\alpha \leq 1$. Let $[A, B] \subset \mathbf{R}, X$ be a Banach space, $f \in C([A, B], X)$. Assume that $f^{\prime}$ exists outside a $\mu$-null Borel set $B_{x} \subseteq[x, B]$, such that

$$
h_{1}\left(f\left(B_{x}\right)\right)=0, \forall x \in[A, B] .
$$

We also assume that $f^{\prime} \in L_{1}([A, B], X)$. Assume $D_{B-}^{\alpha} f \in L_{q}([A, B], X)$. Then

$$
\left\|\frac{1}{B-A} \int_{A}^{B} f(x) d x-f(B)\right\| \leq \frac{\left\|D_{B-}^{\alpha} f\right\|_{L_{q}([A, B], X)}}{\Gamma(\alpha)(p(\alpha-1)+1)^{\frac{1}{p}}\left(\alpha+\frac{1}{p}\right)}(B-A)^{\alpha-\frac{1}{q}} .
$$

We present the following right Caputo-Bochner fractional $L_{q}$ Landau inequality.

Theorem 36. Let $p, q>1: \frac{1}{p}+\frac{1}{q}=1$, and $\frac{1}{q}<\alpha \leq 1$. Let $f \in$ $C^{1}\left(\left(-\infty, B_{0}\right], X\right)$, where $B_{0} \in \mathbf{R}$ is fixed, $X$ is a Banach space. For any $A, B \in\left(-\infty, B_{0}\right]: A \leq B$, we suppose that $f$ fulfills: assume that $f^{\prime \prime}$ exists outside a $\mu$-null Borel set $B_{x} \subseteq[x, B]$, such that

$$
h_{1}\left(f^{\prime}\left(B_{x}\right)\right)=0, \quad \forall x \in[A, B] .
$$

We also assume that $f^{\prime \prime} \in L_{1}([A, B], X)$ and $D_{B-}^{\alpha+1} f \in L_{q}([A, B], X)$. We further assume that

$$
\left\|D_{B-}^{\alpha+1} f\right\|_{L_{q}((-\infty, B], X)} \leq\left\|D_{B_{0}-}^{\alpha+1} f\right\|_{L_{q}\left(\left(-\infty, B_{0}\right], X\right)}<\infty, \quad \forall B \leq B_{0},
$$

(the last left inequality is obvious when $\alpha=1$ ), and

$$
\|f\|_{\infty,\left(-\infty, B_{0}\right]}<\infty
$$




$$
\begin{aligned}
& \text { Then }\left\|f^{\prime}\right\|_{\infty,\left(-\infty, B_{0}\right]} \leq\left(\frac{2\left(\alpha+\frac{1}{p}\right)}{\alpha-\frac{1}{q}}\right)^{\left(\frac{\alpha-\frac{1}{q}}{\alpha+\frac{1}{p}}\right)} \frac{1}{(\Gamma(\alpha))^{\frac{1}{\left(\alpha+\frac{1}{p}\right)}}(p(\alpha-1)+1)^{\frac{1}{(p \alpha+1)}}} . \\
& \left(\|f\|_{\infty,\left(-\infty, B_{0}\right]}\right)^{\left(\frac{\alpha-\frac{1}{q}}{\alpha+\frac{1}{p}}\right)}\left(\left\|D_{B_{0}-}^{\alpha+1} f\right\|_{L_{q}\left(\left(-\infty, B_{0}\right], X\right)}\right)^{\frac{1}{\left(\alpha+\frac{1}{p}\right)}} \cdot(2.141)
\end{aligned}
$$

Proof. By Theorem 35 we have that

$\left\|\frac{1}{B-A} \int_{A}^{B} f^{\prime}(x) d x-f^{\prime}(B)\right\| \leq \frac{\left\|D_{B-}^{\alpha+1} f\right\|_{L_{q}([A, B], X)}}{\Gamma(\alpha)(p(\alpha-1)+1)^{\frac{1}{p}}\left(\alpha+\frac{1}{p}\right)}(B-A)^{\alpha-\frac{1}{q}}$,

$\forall A, B \in\left(-\infty, B_{0}\right]: A \leq B$.

From Theorem 10 we derive

$$
\left\|\frac{f(B)-f(A)}{B-A}-f^{\prime}(B)\right\| \leq \frac{\left\|D_{B-}^{\alpha+1} f\right\|_{L_{q}([A, B], X)}}{\Gamma(\alpha)(p(\alpha-1)+1)^{\frac{1}{p}}\left(\alpha+\frac{1}{p}\right)}(B-A)^{\alpha-\frac{1}{q}},
$$

$\forall A, B \in\left(-\infty, B_{0}\right]: A \leq B$.

Therefore we obtain

$$
\left\|f^{\prime}(B)\right\| \leq \frac{2\|f\|_{\infty,\left(-\infty, B_{0}\right]}}{B-A}+\frac{\left\|D_{B_{0}-}^{\alpha+1} f\right\|_{L_{q}\left(\left(-\infty, B_{0}\right], X\right)}}{\Gamma(\alpha)(p(\alpha-1)+1)^{\frac{1}{p}}\left(\alpha+\frac{1}{p}\right)}(B-A)^{\alpha-\frac{1}{q}},
$$

$\forall A, B \in\left(-\infty, B_{0}\right]: A \leq B$.

The R.H.S. of (2.144) depends only on $B-A$. Therefore

$$
\left\|f^{\prime}\right\|_{\infty,\left(-\infty, B_{0}\right]} \leq \frac{2\|f\|_{\infty,\left(-\infty, B_{0}\right]}}{B-A}+\frac{\left\|D_{B_{0}-}^{\alpha+1} f\right\|_{L_{q}\left(\left(-\infty, B_{0}\right], X\right)}}{\Gamma(\alpha)(p(\alpha-1)+1)^{\frac{1}{p}}\left(\alpha+\frac{1}{p}\right)}(B-A)^{\alpha-\frac{1}{q}} .
$$

We may call $t=B-A>0$. Thus

$$
\left\|f^{\prime}\right\|_{\infty,\left(-\infty, B_{0}\right]} \leq \frac{2\|f\|_{\infty,\left(-\infty, B_{0}\right]}}{t}+\frac{\left\|D_{B_{0}-}^{\alpha+1} f\right\|_{L_{q}\left(\left(-\infty, B_{0}\right], X\right)}}{\Gamma(\alpha)(p(\alpha-1)+1)^{\frac{1}{p}}\left(\alpha+\frac{1}{p}\right)} t^{\alpha-\frac{1}{q}},
$$


$\forall t \in(0, \infty)$.

Notice that $0<\alpha-\frac{1}{q}<1$. Call

$$
\begin{gathered}
\widetilde{\mu}:=2\|f\|_{\infty,\left(-\infty, B_{0}\right]}, \\
\tilde{\theta}:=\frac{\left\|D_{B_{0}-}^{\alpha+1} f\right\|_{L_{q}\left(\left(-\infty, B_{0}\right], X\right)}}{\Gamma(\alpha)(p(\alpha-1)+1)^{\frac{1}{p}}\left(\alpha+\frac{1}{p}\right)},
\end{gathered}
$$

both are positive, and

$$
\widetilde{\nu}:=\alpha-\frac{1}{q} \in(0,1) .
$$

We consider the function

$$
\widetilde{y}(t)=\frac{\widetilde{\mu}}{t}+\widetilde{\theta} t^{\widetilde{\alpha}}, t \in(0, \infty) .
$$

The only critical number here is

$$
\widetilde{t_{0}}=\left(\frac{\widetilde{\mu}}{\widetilde{\alpha} \widetilde{\theta}}\right)^{\frac{1}{\alpha+1}},
$$

and $\widetilde{y}$ has a global minimum at $\widetilde{t_{0}}$, which is

$$
\widetilde{y}\left(\widetilde{t_{0}}\right)=\left(\widetilde{\theta} \widetilde{\mu}^{\widetilde{\alpha}}\right)^{\frac{1}{\alpha+1}}(\widetilde{\alpha}+1) \widetilde{\alpha}^{-\left(\frac{\widetilde{\alpha}}{\alpha+1}\right)} .
$$

Consequently, we derive

$$
\begin{aligned}
& \widetilde{y}\left(\widetilde{t_{0}}\right)=\left(\frac{\left\|D_{B_{0}-}^{\alpha+1} f\right\|_{L_{q}\left(\left(-\infty, B_{0}\right], X\right)}}{\Gamma(\alpha)(p(\alpha-1)+1)^{\frac{1}{p}}\left(\alpha+\frac{1}{p}\right)}\right)^{\frac{1}{\left(\alpha+\frac{1}{p}\right)}} . \\
& \left(2\|f\|_{\infty,\left(-\infty, B_{0}\right]}\right)^{\left(\frac{\alpha-\frac{1}{q}}{\alpha+\frac{1}{p}}\right)}\left(\alpha+\frac{1}{p}\right)\left(\alpha-\frac{1}{q}\right)^{-\left(\frac{\alpha-\frac{1}{q}}{\alpha+\frac{1}{p}}\right)} .
\end{aligned}
$$

We have proved that

$$
\left\|f^{\prime}\right\|_{\infty,\left(-\infty, B_{0}\right]} \leq\left(\frac{2\left(\alpha+\frac{1}{p}\right)}{\alpha-\frac{1}{q}}\right)^{\left(\frac{\alpha-\frac{1}{q}}{\alpha+\frac{1}{p}}\right)} \frac{1}{(\Gamma(\alpha))^{\overline{\left(\alpha+\frac{1}{p}\right)}}(p(\alpha-1)+1)^{\frac{1}{(p \alpha+1)}}} .
$$




$$
\left(\|f\|_{\infty,\left(-\infty, B_{0}\right]}\right)^{\left(\frac{\alpha-\frac{1}{q}}{\alpha+\frac{1}{p}}\right)}\left(\left\|D_{B_{0}-}^{\alpha+1} f\right\|_{L_{q}\left(\left(-\infty, B_{0}\right], X\right)}\right)^{\frac{1}{\left(\alpha+\frac{1}{p}\right)}}
$$

establishing the claim.

Corollary 37. (to Theorem $36, B_{0}=0$ ) All as in Theorem 36 .

Then $\left\|f^{\prime}\right\|_{\infty, \mathbf{R}_{-}} \leq\left(\frac{2\left(\alpha+\frac{1}{p}\right)}{\alpha-\frac{1}{q}}\right)^{\left(\frac{\alpha-\frac{1}{q}}{\alpha+\frac{1}{p}}\right)} \frac{1}{(\Gamma(\alpha))^{\frac{1}{\left(\alpha+\frac{1}{p}\right)}}(p(\alpha-1)+1)^{\frac{1}{(p \alpha+1)}}}$.

Corollary 38. (to Theorem 36, $B_{0}=0, p=q=2$ ) All as in Theorem 36, $\frac{1}{2}<\alpha \leq 1$. Then

$$
\left(\|f\|_{\infty, \mathbf{R}_{-}}\right)^{\left(\frac{\alpha-\frac{1}{q}}{\alpha+\frac{1}{p}}\right)}\left(\left\|D_{0-}^{\alpha+1} f\right\|_{L_{q}\left(\mathbf{R}_{-}, X\right)}\right)^{\frac{1}{\left(\alpha+\frac{1}{p}\right)}} .
$$

$$
\begin{aligned}
& \left\|f^{\prime}\right\|_{\infty, \mathbf{R}_{-}} \leq\left(\frac{2\left(\alpha+\frac{1}{2}\right)}{\alpha-\frac{1}{2}}\right)^{\left(\frac{\alpha-\frac{1}{2}}{\alpha+\frac{1}{2}}\right)} \frac{1}{\frac{1}{(\Gamma(\alpha))^{\left(\alpha+\frac{1}{2}\right)}}(2 \alpha-1)^{\frac{1}{(2 \alpha+1)}}} . \\
& \quad\left(\|f\|_{\infty, \mathbf{R}_{-}}\right)^{\left(\frac{\alpha-\frac{1}{2}}{\alpha+\frac{1}{2}}\right)}\left(\left\|D_{0-}^{\alpha+1} f\right\|_{L_{2}\left(\mathbf{R}_{-}, X\right)}\right)^{\frac{1}{\left(\alpha+\frac{1}{2}\right)}} .
\end{aligned}
$$

Case of $\alpha=1$ follows:

Corollary 39. Let $p, q>1: \frac{1}{p}+\frac{1}{q}=1, f \in C^{1}\left(\left(-\infty, B_{0}\right], X\right)$, where $B_{0} \in \mathbf{R}$ is fixed, $X$ is a Banach space. For any $A, B \in\left(-\infty, B_{0}\right]: A \leq B$, we suppose that $f$ fulfills: assume that $f^{\prime \prime}$ exists outside a $\mu$-null Borel set $B_{x} \subseteq[x, B]$, such that

$$
h_{1}\left(f^{\prime}\left(B_{x}\right)\right)=0, \forall x \in[A, B] .
$$

We further assume that

$f^{\prime \prime} \in L_{q}\left(\left(-\infty, B_{0}\right], X\right)$,

and

$\|f\|_{\infty,\left(-\infty, B_{0}\right]}<\infty$.

Then

$$
\left\|f^{\prime}\right\|_{\infty,\left(-\infty, B_{0}\right]} \leq\left(\frac{2\left(1+\frac{1}{p}\right)}{1-\frac{1}{q}}\right)^{\left(\frac{1-\frac{1}{q}}{1+\frac{1}{p}}\right)}
$$




$$
\left(\|f\|_{\infty,\left(-\infty, B_{0}\right]}\right)^{\left(\frac{1-\frac{1}{q}}{1+\frac{1}{p}}\right)}\left(\left\|f^{\prime \prime}\right\|_{L_{q}\left(\left(-\infty, B_{0}\right], X\right)}\right)^{\frac{1}{\left(1+\frac{1}{p}\right)}} .
$$

Corollary 40. (to Corollary 39) Assume $B_{0}=0$. Then

$$
\left\|f^{\prime}\right\|_{\infty, \mathbf{R}_{-}} \leq\left(\frac{2\left(1+\frac{1}{p}\right)}{1-\frac{1}{q}}\right)
$$

We finish article with

Corollary 41. (to Corollaries 39, 40) Assume $B_{0}=0$ and $p=q=2$. Then

$$
\left\|f^{\prime}\right\|_{\infty, \mathbf{R}_{-}} \leq \sqrt[3]{6}\left(\|f\|_{\infty, \mathbf{R}_{-}}\right)^{\frac{1}{3}}\left(\left\|f^{\prime \prime}\right\|_{L_{2}\left(\mathbf{R}_{-}, X\right)}\right)^{\frac{2}{3}}
$$

Note 42. Many variations and generalizations of the above inequalities are possible, however due to lack of space we stop here.

\section{References}

[1] R.P. Agarwal, V. Lupulescu, D. O'Regan, G. Rahman, Multi-term fractional differential equations in a nonreflexive Banach space, Advances in Difference Equation, (2013), 2013:302.

[2] C.D. Aliprantis and K.C. Border, Infinite Dimensional Analysis, Springer, New York, (2006).

[3] G. Anastassiou, Fractional Differentiation Inequalities, Springer, New York, (2009).

[4] G. Anastassiou, Advances on fractional inequalities, Springer, New York, (2011).

[5] Appendix F, The Bochner integral and vector-valued $L_{p}$-spaces, https://isem.math.kit.edu/images/f/f7/AppendixF.pdf. 
[6] Bochner integral. Encyclopedia of Mathematics. URL: http://www.encyclopediaofmath.org/ index.php?title=Bochner_integral\&oldid=38659.

[7] R.F. Curtain and A.J. Pritchard, Functional Analysis in Modern Applied Mathematics, Academic Press, London, New York, (1977).

[8] M. Kreuter, Sobolev Spaces of Vector-valued functions, Ulm Univ., Master Thesis in Math., Ulm, Germany, (2015).

[9] E. Landau, Einige Ungleichungen für zweimal differentzierban funktionen, Proc. London Math. Soc. 13, pp. 43-49, (1913).

[10] J. Mikusinski, The Bochner integral, Academic Press, New York, (1978).

[11] G.E. Shilov, Elementary Functional Analysis, Dover Publications, Inc., New York, (1996).

[12] C. Volintiru, A proof of the fundamental theorem of Calculus using Hausdorff measures, Real Analysis Exchange, 26 (1), 2000/2001, pp. 381-390.

\section{George A. Anastassiou}

Department of Mathematical Sciences

University of Memphis

Memphis, TN 38152,

U.S.A.

e-mail : ganastss@memphis.edu 